Research Article

\title{
Government-Expert Joint Intervention with Treatment Algorithm and Improved Hypertension Management and Reduced Stroke Mortality in a Primary-Care Setting
}

\author{
Mulalibieke Heizhati $(\mathbb{D}$, Nanfang Li (D), Delian Zhang, Suofeiya Abulikemu, \\ Guijuan Chang, Jing Hong, Nuerguli Maimaiti, Junli Hu, Lei Wang, \\ and Gulinuer Duiyimuhan
}

Hypertension Center of People's Hospital of Xinjiang Uygur Autonomous Region, Xinjiang Hypertension Institute, National Health Committee Key Laboratory of Hypertension Clinical Research China, No. 91 Tianchi Road, Urumqi 830001, Xinjiang, China

Correspondence should be addressed to Mulalibieke Heizhati; morale118@126.com and Nanfang Li; lnanfang2016@sina.com

Received 6 July 2021; Revised 15 September 2021; Accepted 24 September 2021; Published 14 October 2021

Academic Editor: Kai Hu

Copyright ( 2021 Mulalibieke Heizhati et al. This is an open access article distributed under the Creative Commons Attribution License, which permits unrestricted use, distribution, and reproduction in any medium, provided the original work is properly cited.

\begin{abstract}
Hypertension management is suboptimal in the primary-care setting of developing countries, where the burden of both hypertension and cardiovascular disease is huge. Therefore, we conducted a government-expert joint intervention in a resourceconstrained primary setting of Emin, China, between 2014 and 2016, to improve hypertension management and reduce hypertension-related hospitalization and mortality. Primary-care providers were trained on treatment algorithm and physicians for specialized management. Public education was delivered by various ways including door-to-door screening. Program effectiveness was evaluated using screening data by comparing hypertension awareness, treatment, and control rates and by comparing hypertension-related hospitalization and total cardiovascular disease (CVD) and stroke mortality at each phase. As results, 313 primary-health providers were trained to use the algorithm and 3 physicians attended specialist training. 1/3 of locals (49490 of 133376 ) were screened. Compared to the early phase, hypertension awareness improved by $9.3 \%$ ( $58 \%$ vs. $64 \%)$, treatment by $11.4 \%$ (39\% vs. $44 \%)$, and control rates by $33 \%$ (10\% vs. $15 \%)$. The proportion of case/all-cause hospitalization was reduced by $35 \%$ ( $4.02 \%$ vs. $2.60 \%)$ for CVD and by $17 \%$ (3.72\% vs. 3.10\%) for stroke. The proportion of stroke/all-cause death was reduced by $46 \%$ (21.9\% in 2011-2013 vs. $15.0 \%$ in 2014-2016). At the control area, the proportion of case/all-cause mortality showed no reduction. In conclusion, government-expert joint intervention with introducing treatment algorithm may improve hypertension control and decrease related hospitalization and stroke mortality in underresourced settings.
\end{abstract}

\section{Introduction}

About $80 \%$ of mortality from cardiovascular disease (CVD) occurs in low- and middle-income countries [1], mainly due to the increased prevalence of risk factors including elevated blood pressure (BP) and due to relatively lacking access to medical care in these underresourced countries [2]. Hypertension is one of the major risk factors and key drivers for CVD in these countries, especially in underdeveloped regions, whereas it remains undetected, undertreated, and poorly controlled $[3,4]$.
Based on nationwide data, prevalence and mortality rates for ischemic cerebrovascular disease, ischemic heart disease, and hypertensive heart disease in Xinjiang, an underdeveloped province located in Northwestern China, are higher than the national average [5, 6]. Hypertension is responsible for $>70 \%$ of stroke burden in China [6,7]. In Xinjiang, hypertension is affecting $35.0-40.7 \%$ of adults aged $\geq 35$ years $[8,9]$ and $52.6 \%$ of some population [8], higher than national average (41.9\%) [10], whereas the treatment $(28.8 \%$ vs. $34.4 \%)$ and control rates $(10.9 \%$ vs. $15.3 \%)$ are lower $[8,10]$, as in a recent nationwide 
hypertension survey. It showed that control of hypertension in population aged $\geq 18$ years from less-developed regions including Xinjiang is about $8 \%$ [3]. Furthermore, patient-, provider-, and system-related bottlenecks for hypertension control widely exist here $[11,12]$. Furthermore, other risk factors for CVD are highly prevalent such as dyslipidemia, obesity, and high salt intake [13].

Although hypertension in developing countries has been studied substantially, a number of critical problems, especially its proper management, remain unsolved [3]. Given the fact that hypertension plays significant roles in CVD morbidity and mortality $[5,6]$, it is highly needed to undertake interventions to improve hypertension control and to reduce its related morbidity and mortality. Indeed, interventions have been associated with reduction in CVD risk $[4,14]$. In the Kaiser program, the control rate of hypertension increased from $44 \%$ to $90 \%$ and death from stroke fell to $42 \%$ over 13 years of the project [15]. While model interventions provide useful experiences and basis, the ability to deliver satisfactory care can be highly contextualized and there is a lack of knowledge about how to stimulate this kind care in low- and middle-income countries. Therefore, we selected Emin county, a multiethnic lowincome region with constricted medical resource and $>80 \%$ population living in the agricultural and stock-raising region characterized by low education attainment (Table 1), to implement a government-expert joint intervention covering the whole county, involving multiple stakeholders, emphasizing education of primary-care providers and physicians and of the public by various ways such as door-to-door screening, and introducing antihypertensive treatment algorithm between Jan 2014 and Dec 2016. The aim was to raise awareness, treatment and control of hypertension, and to decrease hypertension-related hospitalization and mortality including CVD and stroke at a primary-care setting, and therefore, to explore suitable solutions for areas with similar conditions. In the current report, we evaluated the effects of the hypertension control program after two years.

\section{Methods}

2.1. Context. Intervention area: Emin, in Northern Xinjiang, is home to about 133376 people aged $\geq 15$ years ( $45 \%$ Han and $35 \%$ Kazakh). There were 1 county-level hospital and 22 community health service centers and clinics, with 12 licensed doctors (Table 1).

Control area: Tuoli, a neighbouring county with similar condition about $60 \mathrm{Km}$ away from the Emin county center, was selected as the control area. Adult population aged $\geq 15$ years in Tuoli is about 83106 and comprised of Han (25.3\%) and Kazakh (71.4\%) ethnic groups. There were 1 county hospital, 1 maternity and child-care hospital, and 1 community health service center with 7 stations. Importantly, there were no documented antihypertension programs in Tuoli during this period.

Study protocols were approved by the ethics committee of People's Hospital of Xinjiang Uygur Autonomous Region, China.
2.2. Intervention Design. This is a government-expert joint intervention, involving multiple stakeholders. Depending on previous interventions [15-18] and on local conditions, administration from the top was engaged $[10,11]$. The local government was involved by distributing an official statement and by establishing a cross-sectoral steering committee, efforts were orchestrated from health workers and general public, and input from a central hypertension management team was encompassed.

2.2.1. Concrete Methods Included. (1) Hypertension was set as a priority by distributing an official statement and by establishing a cross-sectoral steering committee; (2) medical staff education; (3) an evidence-based antihypertensive treatment algorithm was introduced to help health-care providers (Figure 1); (4) raising public awareness through various ways including door-to-door screening and public education programs; and (5) contextualizing some parts: materials including books, printed algorithm, and slices were prepared, and training was prepared in 4 languages (Han, Uygur, Kazakh, and Mongolian); considering the cultural and linguistic background, local trusted teachers and hypertensive volunteers delivered health-related information to the general public.

2.3. Education of Primary Health-Care Providers and Physicians. Primary health-care providers including general practitioners and physicians were trained at a 3-day workshop at the start and at a refresh 1-day workshop once 3 months later in 4 languages. Training concentrated on treatment algorithm including components on follow-up guided by algorithm and BP and counselling on healthy life style (Figure 1). Physicians from county-level hospitals were also sent to the hypertension center in the regional tertiary hospital to participate in the training on diagnosis of complicated conditions associated with hypertension to help the referred patients from primary care.

2.4. Public Education. Public education was undertaken together with door-to-door screening by health providers, by wellplanned media (television, newspapers, radio, videos, etc.) and communication messages with a broad range of community activities, involvement of health professionals, volunteers' education (hypertensives and teachers), work site education, gatherings, and handbooks. Contests were conducted regularly at work sites, communities, and among hypertensives as in previous interventions [19].

2.5. Introduction of Treatment Algorithm. Gaps exist between evidence-based recommendations and current practice on hypertension management in developing regions, that health-care providers are less informed or experienced and/or lack willingness to follow guidelines, and or that medications may not be used at the best dosage. Therefore, we introduced treatment algorithm. 
TABle 1: Population characteristics at Emin and Tuoli Xinjiang China.

\begin{tabular}{|c|c|c|}
\hline Characteristics & Emin & Tuoli \\
\hline Population aged $\geq 15$ years $(n)$ & 133376 & 83106 \\
\hline Ethnicity $(n, \%)$ : Han & $60019(45.0)$ & $21025(25.3)$ \\
\hline Kazakh & $46681(35.0)$ & $59337(71.4)$ \\
\hline Uygur & $6669(5.0)$ & $1072(1.29)$ \\
\hline Mongolian & $5335(4.0)$ & - \\
\hline Others & $14671(11.0)$ & $1662(2.0)$ \\
\hline Education $(n, \%)$ : no schooling & $3614(2.71)$ & $2493(3.0)$ \\
\hline Primary & $46148(34.6)$ & $27424(33.0)$ \\
\hline Junior high & $5551(41.5)$ & $37397(45.0)$ \\
\hline Senior high & $17739(13.3)$ & $9972(12.0)$ \\
\hline College or above & $16271(12.2)$ & $5817(7.0)$ \\
\hline Region $(n, \%)$, urban & $44814(33.6)$ & $29087(35.0)$ \\
\hline Agricultural and stock-raising & $10889(81.4)$ & $54019(65.0)$ \\
\hline No. of doctors for per 1000 population and of those with license & 3.1 and 12 & 2.4 and 10 \\
\hline Medical insurance coverage (\%) & \multicolumn{2}{|c|}{$>90 \%$ of population } \\
\hline Gross domestic product in 2014 & \multicolumn{2}{|c|}{ Xinjiang: ranking $25^{\text {th }}$ among 31 provinces } \\
\hline
\end{tabular}

2.6. Rationale for the Treatment Algorithm and Agent Selection. As in Figure 1, we introduced an antihypertensive treatment algorithm with integrative blood pressure (BP) control mechanisms in mind including reduction of sodium and water retention by diuretics, renin aldosterone angiotensin system (RAAS) inhibition by angiotensin-converting enzyme inhibitors (ACEIs), vasodialation by dihydropyridine calcium channel blockers (CCBs), and controlling cardiac rate and output by nondihydropyridine CCB and beta-blockers. We had introduced this algorithm to another three counties of Xinjiang between 1997 and 2017 and observed plausible changes in hypertension management and improvement in related death (just accepted in the International Journal of hypertension). In fact, drug regimens with complementary activity, where a second agent blocks compensatory responses to the initial agent or affects a different pressor mechanism, can result in additive BP lowering [20]. For example, diuretics may stimulate RAAS, and an additive BP-lowering effect may be obtained by adding an ACEI [21]. In addition, to help accelerate clinical decision making, one specific agent (hydroclorothiazide, captopril, nitrendipine, verapamil, and metoprolol) was selected as in the previous program.

2.7. Application of the Treatment Algorithm. Step 1: definition of hypertension: systolic BP (SBP) $\geq 140$ and or diastolic BP (DBP) $\geq 90 \mathrm{mmHg}$ and or known hypertension. Step 2: prescribing hydrochlorothiazide $12.5 \mathrm{mg}$ qd or $25 \mathrm{mg}$ qod and follow-up in 2 weeks. Step 3: adding ACEI or dihydropyridine CCB for another 2 weeks, if $\mathrm{BP} \geq 140 / 90 \mathrm{mmHg}$. Step 4: if BP $\geq 140 / 90 \mathrm{mmHg}$, for the coming 2 weeks, (1) a nondihydropyridine CCB is added if pulse rate $\geq 80 \mathrm{bpm}$ and dihydropyridine CCB if pulse rate $<80 \mathrm{bpm}$ for those on ACEI; (2) a beta-blocker is added if pulse rate $\geq 80 \mathrm{bpm}$ and an ACEI if pulse rate $<80 \mathrm{bpm}$ for those on a dihydropyridine CCB. Step 5: referral to county hospital is recommended if $\mathrm{BP} \geq 140 / 90 \mathrm{mmHg}$ after step 4 . If $\mathrm{BP}$ reached the target level at any phase, follow-up at every 3 months is conducted.
The patients are referred to the next-level hospital if there is a previous heart attack, atrial fibrillation, symptoms/signs of heart failure and known heart failure, chronic kidney disease, symptoms/signs of stroke, known stroke, diabetes, breast-feeding women, pregnant women, women of childbearing age, or BP $>200 / 120 \mathrm{mmHg}$ and $\mathrm{BP}>180$ / $110 \mathrm{mmHg}$ with severe headache, chest pain, breath shortness, blurred vision, or vomiting.

Health-care providers were encouraged to follow the algorithm at the population screening and at their regular clinical work unless clinical discretion required for the abovementioned conditions, since in settings where people do not regularly visit the doctor, people who are just recommended only lifestyle modification may not return for reevaluation and needed treatment, resulting in uncontrolled hypertension and associated complications [22]. For managing known hypertension, health-care providers were advised to review medication and BP; simplify regimens; or change to the antihypertensive treatment algorithm of this program. Recommended target BP was $<140 / 90 \mathrm{mmHg}$.

Health-care providers were also educated to provide information to patients on the importance of long-term medication intake, even if there are no symptoms, on how to take medications at home, to explain how many times, and at what time to take medication; explain potential adverse effects; and what to do if patients experience.

2.8. Door-to-Door Screening. Health workers performed door-to-door screening and public education to eligible residents since 1 Jan 2014 to 31 Dec 2016. The eligible population included locals aged $\geq 15$ years. Informed consent was obtained from all subjects and from the parents or legal guardians for those under 18 years of age.

2.9. Data Collection. In Emin, anthropometric and clinical measurements using questionnaires were taken during door-to-door screening. Questions assessed demographic characteristics, education, lifestyle risk factors (cigarette and alcohol use), and hypertension-related 


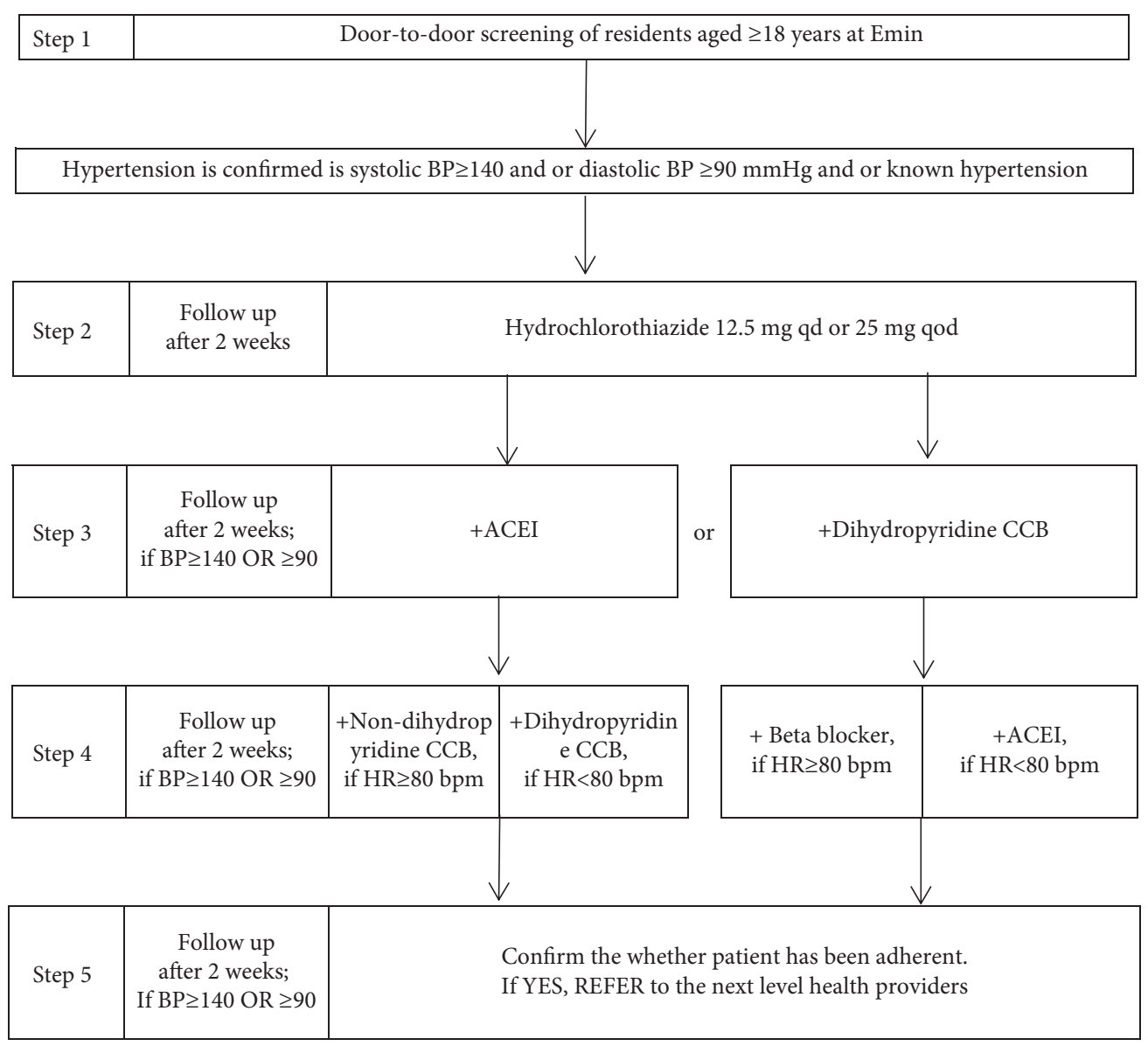

Healthy life style tips

Restrict salt to $<6$ grams per day gradually ( 1 teaspoon per day); Limit use of fatty food, specially fatty meat, dairy fat and cooking oil; Limit meat to once to twice a week; Increase fruits and vegetables intake ( 5 servings per day); Increase activity (brisk walk $\geq 150$ min per week); Lose weight, if overweight or obese; Stop smoking; Avoid alcohol

Refer to next level, if:

a previous heart attack, symptoms/signs of stroke and known stroke,atrial fibrillation, Symptoms/signs of heart failure, and known heart failure, chronic kidney disease, diabetes mellitus, breast-feeding women, pregnant women, women of childbearing age, $\mathrm{BP}>200 / 120 \mathrm{mmHg}$, or $\mathrm{BP}>180 / 110 \mathrm{mmHg}$ with severe headache, chest pain, breath shortness, blurred vision,or vomiting.

FIGURE 1: Hypertension treatment algorithm.

information. Physical examination including BP measurement was conducted as well. We measured BP three times using an electronic sphygmomanometer (Omron HEM-7201) from the unclothed right arm of the person in a sitting position at intervals of at least $1 \mathrm{~min}$ after a rest of at least $5 \mathrm{~min}$. Mean of the three BP was used in this analysis. Body weight, height, and waist circumference were measured using standard methods. Body mass index (BMI) was calculated as body weight divided by squared height $\left(\mathrm{kg} / \mathrm{m}^{2}\right)$.

In addition, we collected data on hospitalization and hospital stay length for hypertension, CVD, and stroke from the medical insurance system and county hospitals both in the intervention and control area between 2014 and 2016. 
Furthermore, we collected death data (death diagnosis and certificate) from the hospital information and household registration system of both the intervention and control area between 2011 and 2016. However, we did not collect BP- and hypertension-related data from the population in the control area.

2.10. Grouping and Evaluation. To evaluate the overall impact of the intervention, first, we divided study participants into three groups as early-, middle-, and later-phase intervention groups, based on the time of being screened (numbered by the screening date and tertiled), and compared the awareness, treatment, and control of hypertension and BP levels among participants in early-, middle-, and later-phase groups. Second, we compared the proportion of case/all-cause hospitalization and hospital stay length for hypertension, CVD, and stroke among participants in early-, middle-, and later-phase groups, using the main diagnosis at hospital discharge. Third, we compared the proportion of case/all-cause death for CVD and stroke using death diagnosis on the death certificate from the hospital information and household registration system at the intervention (Emin) and control (Tuoli) area before (2011-2013) and during the intervention (2014-2016).

2.11. Statistical Analysis. Continuous data such as age, BMI, waist circumference, and SBP and DBP were expressed as mean \pm SD if normally distributed and compared using the ANOVA test followed by the post hoc analysis (LSD) test among the groups. Categorical data including gender composition, ethnic groups, education attainments, living area, and smoking and drinking status were expressed as $(n, \%)$ and compared using the $X^{2}$ test among the groups where necessary. Prevalence, awareness, treatment, and control rates of hypertension were calculated, expressed as $\%, 95 \%$ confidence interval, and compared using the $X^{2}$ test among groups. The proportion of case/all-cause hospitalization and of case/all-cause hospital stay for hypertension, CVD, and stroke and the proportion of case/all-cause mortality for CVD and stroke were calculated, expressed as $\%$, and compared using the $X^{2}$ test among the groups. SPSS software version 19 was used for statistical analysis, and $P<0.05$ was considered statistically significant.

\section{Results}

3.1. Activity Metrics. During the intervention, 313 healthcare providers were trained for the algorithm once 3 months and 3 physicians attended training at the regional hypertension center for 3 months each time.

3.2. Statisticsfor Screening Subjects. During the door-to-door visit, 59405 subjects were counseled on healthy life and invited for screening, of whom 49497 subjects (response rate: $83.3 \%)$ were screened, covering $37.1 \%$ (49497/133376) of adult residents, and there were complete data for 47040 subjects.
The early-, middle-, and later-phase subjects were similar in age and gender composition, whereas the later-phase subjects were less smokers and drinkers and had lower BMI and waist circumference (Table 2).

Compared to the early phase, awareness, treatment, and control rates improved by $9.3 \%$ (early vs. later phase: $58 \%$ vs. $64 \%$ ), $11.4 \%$ (39\% vs. $44 \%$ ), and $33 \%$ (10\% vs. $15 \%)$. The control rate of treated hypertension improved by $31.9 \%$ (20.4 vs. $27 \%$ ) (Table 3 ).

Compared to the early phase, total hypertensives (146.9 vs. 144.4 vs. $144.1 \mathrm{mmHg}$ ) and hypertensives with awareness (145.2 vs. 143.9 vs. $143.4 \mathrm{mmHg}$ ) and under treatment (143.9 vs. 141.7 vs. $141.5 \mathrm{mmHg}$ ) at the middle and later phase showed significant lower SBP levels.

In addition, total population and total hypertensives showed lower DBP levels at the later phase, compared to the early phase (77.4 vs. $76.7 \mathrm{mmHg}$ and 89.3 vs. $87.8 \mathrm{mmHg}$, respectively, Table 4 ).

3.3. Statistics on Hypertension-Related Hospitalization and Death Data. Compared to the early phase, the proportion of case/all-cause hospitalization reduced by $35.3 \%$ ( $4.02 \%$ vs. $2.60 \%$ ) for total CVD and by $16.6 \%$ (3.72\% vs. $3.10 \%)$ for stroke (Table 5). The proportion of case/total hospital stay reduced by $32.5 \%$ ( 4.34 vs. $2.93 \%$ ) for CVD and by $10.8 \%$ (4.46 vs. $3.98 \%$ ) for stroke (Table 5).

Compared to 2011-2013, the proportion of total CVD/ all-cause death and of stroke/all-cause death showed a decreasing trend in 2014-2016 at Emin county. The proportion of CVD/all-cause death reduced by $17 \%$ (33\% vs. $27.4 \%$, statistically nonsignificant), and that of stroke/all-cause death reduced by $31.5 \%(21.9 \%$ vs. $15.0 \%, P<0.001)$ in 2014-2016.

In the control area, the proportion of total CVD and stroke/all-cause death showed a minor but not significant decrease during the same period (Table 6).

\section{Discussion}

Hypertension is a key CVD driver and remains widely undetected, undertreated, and poorly controlled, especially in underdeveloped areas $[3,4]$. This is a government-expert joint program with tranings of health-care providers and introduction of an algorithm. Main results encompass awareness, treatment, and control rates for hypertension improved by $9.3 \%, 11.4 \%$, and $33 \%$ at the later phase than the early phase. The proportion of case/all-cause hospitalization reduced by $17.3 \%$ for hypertension, by $35 \%$ for CVD, and by $17 \%$ for stroke. The proportion of stroke/all-cause death was reduced by $31.5 \%$.

At the early phase, $>40 \%$ of hypertensives remain unaware, $>60 \%$ untreated, and $>90 \%$ BP uncontrolled, possibly indicating major bottlenecks for ineffective control are diagnosis, effective screening, and treatment titration. Hence, screening and quality improvement to ensure titration of medications may be critical for better outcomes [18]. After 2 years, awareness reached $64 \%$, higher than national average (46.9\%) [3]. Raising awareness is the first step for successful 
TABLE 2: Characteristics of the population screened at the early, middle, and later phase.

\begin{tabular}{|c|c|c|c|c|c|c|c|}
\hline Characteristics & Early phase & Middle phase & Later phase & $P 1$ & $P 2$ & $P 3$ & $P$ \\
\hline Total $(n)$ & 15680 & 15680 & 15680 & & & & \\
\hline Gender (men, $n, \%)$ & $8052(51.4)$ & $11295(48.5)$ & $8157(52.0)$ & - & - & - & 0.015 \\
\hline Age (years) & $43.6 \pm 15.1$ & $44.7 \pm 15.5$ & $43.4 \pm 15.4$ & $<0.001$ & 0.289 & $<0.001$ & $<0.001$ \\
\hline Body mass index $\left(\mathrm{kg} / \mathrm{m}^{2}\right)$ & $24.6 \pm 3.4$ & $24.6 \pm 3.4$ & $24.4 \pm 3.5$ & - & 0.028 & 0.016 & $<0.001$ \\
\hline Waist circumference $(\mathrm{cm})$ & $85.1 \pm 11.5$ & $85.3 \pm 12.4$ & $84.8 \pm 11.6$ & - & 0.002 & $<0.001$ & $<0.001$ \\
\hline Smoking status $(n, \%)$ & $2321(14.8)$ & $3242(13.9)$ & $1902(12.1)$ & - & - & - & 0.002 \\
\hline Drinking status $(n, \%)$ & $2965(18.9)$ & $4287(18.4)$ & $2458(15.7)$ & - & - & - & $<0.001$ \\
\hline
\end{tabular}

$P 1$ : early vs. middle phase; $P 2$ : early vs. later phase; $P 3$ : middle vs. later phase; $P$ : for among-group comparison.

TABle 3: Awareness, treatment, and control rates of hypertension at the early, middle, and later phase (\%, 95\% CI).

\begin{tabular}{lcccc}
\hline & Early phase & Middle phase & Later phase & \multicolumn{1}{c}{$X^{2} / P$} \\
\hline Total subjects $(n)$ & 15680 & 15680 & 15680 & 4615 \\
Hypertensive patients $(n)$ & 4030 & 4265 & $29.3(29.2,30.3)$ & $54.65 /<0.001$ \\
Age-standardized prevalence & $25.9(25.2,26.6)$ & $27.2(26.3,28.1)$ & $64.0(63.3,66.1)$ & $32.67 /<0.001$ \\
Awareness of hypertension & $58.0(56.0,59.0)$ & $66.7(66.1,68.0)$ & $44.2(43.1,45.2)$ & $22.14 /<0.001$ \\
Treatment of hypertension & $39.0(38.1,41.0)$ & $46.1(45.0,47.5)$ & $68.6(67.0-70.0)$ & $2.34 / 0.834$ \\
Treatment of in the aware & $67.5(65.2,69.1)$ & $68.0(67.2,69.2)$ & $15.0(14.1,16.2)$ & $48.52 /<0.001$ \\
Control of hypertension & $10.0(9.1,11.0)$ & $17.1(16.2,18.5)$ & $27.0(26.5,28.4)$ & $30.32 /<0.001$ \\
Control of treated hypertension & $20.4(19.3,21.3)$ & $29.4(29.1,31.5)$ & & \\
\hline
\end{tabular}

95\% CI: 95\% confidence interval.

TABLE 4: Blood pressure levels in study participants at the early, middle, and later phase.

\begin{tabular}{|c|c|c|c|c|c|c|c|}
\hline & Early phase & Middle phase & Later phase & $P 1$ & $P 2$ & $P 3$ & $P$ \\
\hline \multicolumn{8}{|c|}{ Systolic blood pressure (mmHg) } \\
\hline Total & $123.8 \pm 17.60$ & $121.9 \pm 16.8$ & $123.2 \pm 15.4$ & $<0.001$ & - & $<0.001$ & \\
\hline Hypertensives & $146.9 \pm 16.1$ & $144.4 \pm 13.4$ & $144.1 \pm 11.1$ & $<0.001$ & $<0.001$ & 0.047 & $<0.001$ \\
\hline Aware & $145.2 \pm 14.4$ & $143.9 \pm 12.3$ & $143.4 \pm 10.7$ & $<0.001$ & $<0.001$ & 0.046 & $<0.001$ \\
\hline Unaware & $145.9 \pm 10.5$ & $145.1 \pm 14.5$ & $146.4 \pm 8.5$ & - & - & - & - \\
\hline Treated & $143.9 \pm 16.1$ & $141.7 \pm 11.7$ & $141.5 \pm 14.9$ & $<0.001$ & $<0.001$ & - & $<0.001$ \\
\hline Untreated & $147.8 \pm 12.1$ & $147.1 \pm 17.7$ & $148.1 \pm 12.7$ & - & - & - & - \\
\hline Controlled & $131.4 \pm 6.7$ & $131.4 \pm 7.1$ & $132.2 \pm 6.4$ & - & - & - & - \\
\hline Uncontrolled & $144.6 \pm 12.5$ & $146.1 \pm 16.1$ & $144.6 \pm 12.0$ & $<0.001$ & - & $<0.001$ & \\
\hline \multicolumn{8}{|c|}{ Diastolic blood pressure (mmHg) } \\
\hline Total & $77.4 \pm 10.3$ & $76.6 \pm 9.8$ & $76.7 \pm 9.1$ & - & - & - & - \\
\hline Hypertensives & $89.3 \pm 9.7$ & $89.2 \pm 6.9$ & $87.8 \pm 8.5$ & - & $<0.001$ & $<0.001$ & $<0.001$ \\
\hline Aware & $88.1 \pm 7.6$ & $87.9 \pm 10.3$ & $88.0 \pm 9.3$ & $<0.001$ & 0.793 & $<0.001$ & $<0.001$ \\
\hline Unaware & $80.3 \pm 7.8$ & $80.9 \pm 9.5$ & $84.5 \pm 5.3$ & - & $<0.001$ & $<0.001$ & $<0.001$ \\
\hline Treated & $88.4 \pm 8.4$ & $88.4 \pm 9.5$ & $89.3 \pm 7.4$ & - & - & - & - \\
\hline Untreated & $84.2 \pm 9.1$ & $85.2 \pm 11.1$ & $86.5 \pm 6.7$ & - & - & $<0.001$ & - \\
\hline Controlled & $81.6 \pm 6.0$ & $81.5 \pm 6.4$ & $82.9 \pm 4.7$ & - & - & - & - \\
\hline Uncontrolled & $86.3 \pm 9.2$ & $88.4 \pm 10.4$ & $88.5 \pm 7.3$ & $<0.001$ & $<0.001$ & - & $<0.001$ \\
\hline
\end{tabular}

$P 1$ : early vs. middle phase; $P 2$ : early vs. later phase; $P 3$ : middle vs. later phase; $P$ : among-group comparison.

hypertension management, and the better way is screening [4]. Door-to-door screening and public education may have benefited the population by increasing health awareness [23]. Treatment and control rates are $44 \%$ and $15 \%$ at the later phase, comparable to the national average $(40.7 \%$ and $15.3 \%$ ) [3] and those of many parts of the world (13\%) [24]. One important aspect may be the involvement of the local government. Another critical part possibly is the algorithm as put forward in the WHO HEARTS program [22]. Its presence may have helped clinical decision making by addressing the difficulty in choosing medicine $[10,11]$. Additionally, an agent was recommended from each type, which may have facilitated agent prescription.
At the later phase, the program achieved some success in hypertension management, reflected by improved treatment and control rates, and by the decreased hypertension-related hospitalization and hospital stay. In fact, better out-ofhospital BP control has a positive effect on reducing hospitalization [25-28]. The proportion of stroke/all-cause death during the intervention was reduced by $31.5 \%$. The program may have helped capacity building on hypertension management, followed by decreased stroke mortality through better out-of-hospital BP control, reduction in stroke incidence, and improvement in its survival [29]. While it is difficult to provide a comprehensive stroke mortality analysis in regional programs, we have tried to 
TABLE 5: Changes in the proportion of case/total hospitalization and of case hospital stay days for hypertension, CVD, and stroke at the early, middle, and later phase at the intervention area.

\begin{tabular}{|c|c|c|c|c|}
\hline & Early phase & Middle phase & Later phase & $P$ \\
\hline \multicolumn{5}{|l|}{ All-cause } \\
\hline Subjects $(n)$ & 18258 & 18122 & 21083 & \\
\hline Total hospital stay (days) & 165023 & 161084 & 185519 & \\
\hline \multicolumn{5}{|l|}{ Hypertension } \\
\hline Subjects $(n)$ & 1852 & 1586 & 1769 & \\
\hline Total hospital stay (days) & 17800 & 15012 & 16974 & \\
\hline Average hospital stay (days) & 9.61 & 9.47 & 9.60 & \\
\hline$\%$ of case/total hospitalization & 10.14 & 8.75 & 8.39 & $<0.001$ \\
\hline$\%$ of case/total hospital stay & 10.79 & 9.32 & 9.15 & $<0.001$ \\
\hline \multicolumn{5}{|l|}{ Total CVD } \\
\hline Subjects $(n)$ & 735 & 663 & 548 & \\
\hline Total hospital stay (days) & 77169 & 6492 & 5430 & \\
\hline Average hospital stay (days) & 9.75 & 9.79 & 9.91 & \\
\hline$\%$ of case/total hospitalization & 4.02 & 3.65 & 2.60 & $<0.001$ \\
\hline$\%$ of case/total hospital stay & 4.34 & 4.03 & 2.93 & $<0.001$ \\
\hline \multicolumn{5}{|l|}{ Total stroke } \\
\hline Subjects $(n)$ & 680 & 624 & 654 & \\
\hline Total hospital stay (days) & 7361 & 6661 & 7390 & \\
\hline Average hospital stay (days) & 10.83 & 10.68 & 11.30 & \\
\hline$\%$ of case/total hospitalization & 3.72 & 3.44 & 3.10 & $<0.001$ \\
\hline$\%$ of case/total hospital stay & 4.46 & 4.14 & 3.98 & $<0.001$ \\
\hline
\end{tabular}

CVD: cardiovascular disease.

TABLE 6: Changes in the proportion of case/all-cause death for total CVD and stroke in intervention and control before and during the intervention period.

\begin{tabular}{|c|c|c|c|}
\hline & $2011-2013$ & $2014-2016$ & $X^{2} / P$ \\
\hline \multicolumn{4}{|l|}{ Emin (intervention area) } \\
\hline All-cause death $(n)$ & 1258 & 1298 & \\
\hline Total CVD death $(n)$ & 415 & 355 & \\
\hline Proportion of CVD in all-cause death (\%) & 33.0 & 27.4 & $1.14 / 0.684$ \\
\hline Stroke death $(n)$ & 276 & 195 & \\
\hline Proportion of stroke in all-cause death (\%) & 21.9 & 15.0 & $16.78 /<0.001$ \\
\hline \multicolumn{4}{|l|}{ Tuoli (control area) } \\
\hline All-cause death $(n)$ & 1554 & 2004 & \\
\hline Total CVD death $(n)$ & 533 & 653 & \\
\hline Proportion of CVD in all-cause death (\%) & 34.3 & 32.6 & $1.87 / 0.188$ \\
\hline Stroke death $(n)$ & 347 & 435 & \\
\hline Proportion of stroke in all-cause death (\%) & 22.3 & 21.7 & $0.001 / .0997$ \\
\hline
\end{tabular}

CVD: cardiovascular disease.

explore this by including the data with comparable regions. Unlike Emin, the hypertension program was not conducted at Tuoli, where the death proportion for CVD including stroke was not changed much. Although these data may not establish causality, an expected pattern of correlation between the program and stroke mortality composition is observed in the intervention county.

Prevalence, awareness, treatment, and control of hypertension were $26 \%, 58 \%, 39 \%$, and $10 \%$ at the start. Prevalence and awareness were higher than the national average (23.2\% and 46.9\%) [3], possibly driven by the difference in survey methods. The population with health problems is more prone to accept screening and have increased health concerns, which may have some relevance with higher prevalence and awareness. Also, Kazakhs and
Mongolians with higher hypertension prevalence $[8,9,30]$ account for $39 \%$ of the study population. Control rate at the early phase $(10 \%)$ was comparable to the $8 \%$ for less-developed areas in the national data [3] and comparable to the national average at the later phase (15.0\% vs. $15.3 \%)$ [3]. Improvements have been more ideal if individuals combated hypertension through lifestyle and followed up [31].

Current analysis is strengthened by subjects covering over one-third of locals and by their diversity in age, sex, and ethnicity, which may make them representative of locals [32]. Nonetheless, several points deserve consideration when interpreting the data. First, the before-after comparison design does not allow us to establish causal relation between the program and observed plausible changes. Results would have been more ideal if the same subjects were included, 
whereas a cross-sectional approach is a common way [33]. In addition, it was aimed to raise public awareness. Second, effects of counselling on healthy life style and patient compliance were not evaluated. However, beneficial changes in BP might suggest the success in part. Furthermore, lack of a control area should also be kept in mind in terms of secular trends. Nevertheless, we addressed this element by comparing changes in the proportion of CVD and stroke observed in Emin county to the neighboring Tuoli county. In addition, results just after the 2-year time may not be affected by the secular trends, based on similar examples [34]. Finally, we failed to collect the data on the average time to reach the target $\mathrm{BP}$ and the number of agents taken by the patients at the time of target $\mathrm{BP}$, which are the important indicators to assess the efficiency and effectiveness of an algorithm. However, previous studies from communitybased patients of China reported that treating hypertension with nitrendipine and hydrochlorothiazide and with nitrendipine and metoprolol will allow more reasonable and efficient allocation of limited resources in low-income countries [35].

In conclusion, government-expert joint intervention with the introduction of the treatment algorithm may help capacity building on hypertension management and decrease hypertension-related CVD morbidity and mortality at a medical resource-constricted primary setting with low income and education.

\section{Data Availability}

Data can be made available on reasonable request.

\section{Conflicts of Interest}

The authors declare no conflicts of interest.

\section{Authors' Contributions}

Mulalibieke Heizhati and Nanfang Li contributed equally to this work. NF L put forward, designed, and implemented the intervention, analyzed the data, and drafted the manuscript. M H, L Z, DL Z, S A, GJ C, J H, N M, J H, L W, and G D participated in the intervention design and intervention implementation, data analysis and interpretation, and the manuscript writing.

\section{Acknowledgments}

The authors thank all the study participants for supporting the program and thank volunteers for providing great help. They also thank county leaders and leaders of the health, education, finance, and science and technology bureau for their greatest support in this program and making hypertension as the priority and for establishing a committee to conduct and oversee the intervention. Finally, they thank the Local and National Department of Science and Technology for funding the project. This program was funded by the Ministry of Science and Technology of China (2013GS650101).

\section{References}

[1] Global Status Report on Noncommunicable Diseases 2010, World Health Organization, Geneva, Switzerland, 2011.

[2] S. Yusuf, S. Rangarajan, K. Teo et al., "Cardiovascular risk and events in 17 low-, middle-, and high-income countries," New England Journal of Medicine, vol. 371, no. 9, pp. 818-827, 2014.

[3] Z. Wang, Z. Chen, L. Zhang et al., "Status of hypertension in China," Circulation, vol. 137, no. 22, pp. 2344-2356, 2018.

[4] A Global Brief on Hypertension, World Health Organization, Geneva, Switzerland, 2013.

[5] M. Zhou, H. Wang, J. Zhu et al., "Cause-specific mortality for 240 causes in China during 1990-2013: a systematic subnational analysis for the global burden of disease study 2013," The Lancet, vol. 387, no. 10015, pp. 251-272, 2016.

[6] W. Wang, B. Jiang, H. Sun et al., "Prevalence, incidence, and mortality of stroke in China," Circulation, vol. 135, no. 8, pp. 759-771, 2017.

[7] M. Liu, B. Wu, W.-Z. Wang, L.-M. Lee, S.-H. Zhang, and L.-Z. Kong, "Stroke in China: epidemiology, prevention, and management strategies," The Lancet Neurology, vol. 6, no. 5, pp. 456-464, 2007.

[8] Y.-T. Wang, D. Adi, Z.-X. Yu et al., "The burden and correlates of hypertension among Chinese rural population in Han, Uygur, and Kazak: a cross-sectional study," Journal of the American Society of Hypertension, vol. 11, no. 11, pp. 737-745, 2017.

[9] J. Jiang, B. Zhang, M. Zhang et al., "Prevalence of conventional cardiovascular disease risk factors among Chinese Kazakh individuals of diverse occupational backgrounds in Xinjiang China," International Journal of Cardiology, vol. 179, pp. 558-560, 2015.

[10] W. Li, H. Gu, K. K. Teo et al., "Hypertension prevalence, awareness, treatment, and control in 115 rural and urban communities involving 47000 people from China," Journal of Hypertension, vol. 34, no. 1, pp. 39-46, 2016.

[11] S. Basu and C. Millett, "Social epidemiology of hypertension in middle-income countries," Hypertension, vol. 62, no. 1, pp. 18-26, 2013.

[12] S. MacMahon, M. H. Alderman, L. H. Lindholm, L. Liu, R. A. Sanchez, and Y. K. Seedat, "Blood-pressure-related disease is a global health priority," The Lancet, vol. 371, no. 9623, pp. 1480-1482, 2008.

[13] N. Li, H. Wang, Z. Yan, X. Yao, J. Hong, and L. Zhou, "Ethnic disparities in the clustering of risk factors for cardiovascular disease among the Kazakh, Uygur, Mongolian and Han populations of Xinjiang: a cross-sectional study," BMC Public Health, vol. 12, no. 1, p. 499, 2012.

[14] World Health Organization Executive Board, Draft Action Plan for the Prevention and Control of Noncommunicable Diseases 2013-2020.11 January 2013, World Health Organization Executive Board, Geneva, Switzerland, 2013, http:// apps.who.int/gb/ebwha/pdf_files/EB132/B132_7-en.pdf.

[15] M. G. Jaffe and J. D. Young, "The Kaiser permanente Northern California story: improving hypertension control from $44 \%$ to 90\% in 13 Years (2000 to 2013)," Journal of Clinical Hypertension, vol. 18, pp. 260-261, 2016.

[16] M. Mozheyko, S. Eregin, N. Danilenko et al., "Hypertension in Russia: changes observed after 4 years of a comprehensive health system improvement program in the yaroslavl region," The Journal of Clinical Hypertension, vol. 19, no. 2, pp. 198204, 2017. 
[17] A. Nissinen, X. Berrios, and P. Puska, "Community-based noncommunicable disease interventions: lessons from developed countries for developing ones," Bulletin of the World Health Organization, vol. 79, pp. 963-970, 2001.

[18] S. O. Oti, S. Van De Vijver, G. B. Gomez et al., "Outcomes and costs of implementing a community-based intervention for hypertension in an urban slum in Kenya," Bulletin of the World Health Organization, vol. 94, no. 7, pp. 501-509, 2016.

[19] J. L. O'Loughlin, G. Paradis, K. Gray-Donald, and L. Renaud, "The impact of a community-based heart disease prevention program in a low-income, inner-city neighborhood," American Journal of Public Health, vol. 89, no. 12, pp. 1819-1826, 1999.

[20] A. H. Gradman, J. N. Basile, B. L. Carter, and G. L. Bakris, "Combination therapy in hypertension," The Journal of Clinical Hypertension, vol. 13, no. 3, pp. 146-154, 2011.

[21] P. K. Whelton, R. M. Carey, W. S. Aronow et al., "2017ACC/ AHA/AAPA/ABC/ACPM/AGS/APhA/ASH/ASPC/NMA/ PCNA guideline for the prevention, detection, evaluation, and management of high blood pressure in adults," $\mathrm{Hy}$ pertension, vol. 71, no. 6, pp. e116-135, 2018.

[22] Hearts Technical Package for Cardiovascular Disease Management in Primary Health Care: Healthy-Lifestyle Counselling, World Health Organization, Geneva, Switzerland, 2018.

[23] M. G. Jaffe, G. A. Lee, J. D. Young, S. Sidney, and A. S. Go, "Improved blood pressure control associated with a largescale hypertension program," JAMA, vol. 310 , no. 7 , pp. 699-705, 2013.

[24] C. K. Chow, K. K. Teo, S. Rangarajan et al., "Prevalence, awareness, treatment, and control of hypertension in rural and urban communities in high-, middle-, and low-income countries," Journal of the American Medical Association, vol. 310, no. 9, pp. 959-968, 2013.

[25] E. C. Yiannakopoulou, J. S. Papadopulos, D. V. Cokkinos, and T. D. Mountokalakis, "Adherence to antihypertensive treatment: a critical factor for blood pressure control," European Journal of Cardiovascular Prevention and Rehabilitation: Official Journal of the European Society of Cardiology, Working Groups on Epidemiology \& Prevention and Cardiac Rehabilitation and Exercise Physiology, vol. 12, pp. 243-249, 2005.

[26] W. J. Elliott, "Improving outcomes in hypertensive patients: focus on adherence and persistence with antihypertensive therapy," The Journal of Clinical Hypertension, vol. 11, no. 7, pp. 376-382, 2009.

[27] N. S. Breekveldt-Postma, F. J. A. Penning-van Beest, S. J. Siiskonen et al., "Effect of persistent use of antihypertensives on blood pressure goal attainment," Current Medical Research and Opinion, vol. 24, no. 4, pp. 1025-1031, 2008.

[28] M. C. Sokol, K. A. McGuigan, R. R. Verbrugge, and R. S. Epstein, "Impact of medication adherence on hospitalization risk and healthcare cost," Medical Care, vol. 43, no. 6, pp. 521-530, 2005.

[29] J. Hata, T. Ninomiya, Y. Hirakawa et al., "Secular trends in cardiovascular disease and its risk factors in Japanese," Circulation, vol. 128, no. 11, pp. 1198-1205, 2013.

[30] R. Yan, W. Li, L. Yin, Y. Wang, and J. Bo, "Cardiovascular diseases and risk-factor burden in urban and rural communities in high-, middle-, and low-income regions of China: a large community-based epidemiological study," Journal of the American Heart Association, vol. 6, p. e004445, 2017.

[31] D. Li, J. Lv, F. Liu et al., "Hypertension burden and control in mainland China: analysis of nationwide data 2003-2012," International Journal of Cardiology, vol. 184, pp. 637-644, 2015.
[32] R. W. Yeh, S. Sidney, M. Chandra, M. Sorel, J. V. Selby, and A. S. Go, "Population trends in the incidence and outcomes of acute myocardial infarction," New England Journal of Medicine, vol. 362, no. 23, pp. 2155-2165, 2010.

[33] R. Cífková, Z. Škodová, J. Bruthans et al., "Longitudinal trends in major cardiovascular risk factors in the Czech population between 1985 and 2007/8. Czech MONICA and Czech postMONICA," Atherosclerosis, vol. 211, no. 2, pp. 676-681, 2010.

[34] N. Sarrafzadegan, R. Kelishadi, A. Esmaillzadeh et al., "Do lifestyle interventions work in developing countries? findings from the Isfahan healthy heart program in the Islamic Republic of Iran," Bulletin of the World Health Organization, vol. 87, no. 1, pp. 39-50, 2009.

[35] Z. Wang, Z. Chen, X. Wang et al., "Cost-effectiveness of nitrendipine and hydrochlorothiazide or metoprolol to treat hypertension in rural community health centers in China," Journal of Hypertension, vol. 35, no. 4, pp. 886-892, 2017. 